\title{
A NOTE ON THE THEORY OF SOCIAL CHOICE
}

Professor Arrow brings to his treatment of the theory of social welfare (1) a fine unity of mathematical rigour and insight into fundamental issues of social philosophy. The problem is the old one of the relationship of individual values to the general well-being of the group.

Arrow eschews any concept of a utility measure which could be validly employed in inter-personal comparison of aggregation. The justification for doing so lies, of course, in the fact that if such a measure is not operationally undefinable, at least it has never been operationally defined. In common with other theorists of the "new" welfare economics, Arrow must be content to fall back on the purely ordinal properties of personal preferences.

These ordinal properties are defined by two simple axioms: I. Given any two alternatives, each individual can state that a particular one of them is at least as good as the other. (If one of the alternatives is judged to be better than the other, it is, perforce, at least as good as the other. If the two alternatives are judged to be indifferent, each is at least as good as the other.) II. Given any three alternatives, if the first is at least as good as the second, and the second at least as good as the third, the individual will judge the first at least as good as the third.

The force of these two axioms together is simply that, given any number of alternatives, each individual is able to arrange them in an array such that each alternative is at least as good as any that follows it and no better than any that preceeds it. (When the individual is indifferent among several alternatives, their position in the array vis-à-vis one another has, of course, no significance since any arrangement of them will satisfy the conditions above.)

Arrow defines a social welfare function as a function defined on the system of all sets of individual arrays carrying each set of indi-

(1) Kenneth J. Arrow, Social Choice and Individual Values. Cowles Commission Monograph No. 12, John Wiley and Sons, Inc. New York, Chapman and Hall Ltd., London, 1951. 
vidual arrays into a social array. That is, the social welfare function defines a rule, or method, whereby given the several orderings of possible alternatives made by the several individuals of a society, a social ordering, having the properties of the axioms, is determined.

The alternatives, the ranking of which Arrow considers, are "social states" in the broadest sense of the term. Moreover, in ranking these alternative social states, individuals are free to consider not only the amounts and kinds of services produced, the particulars of the distribution of these services among the members of the community, but may also consider any difference whatever that happens to interest them. Thus the problem of the social welfare function is a generalization of the problem of welfare economics, including the whole range of social decisions in its scope.

If nothing more is required of the social welfare function than what has been outlined above, the construction of social welfare functions is an easy matter indeed. For example, nothing has been said about the number of possible alternatives. If there are only two alternatives, the method of majority vote provides a perfectly good social welfare function. However, if there are more than two distinct social states majority vote does not necessarily work. Arrow illustrates this point by his "paradox of voting": suppose there are three individuals $(1,2,3)$ and three alternatives $(X, Y, Z)$. Suppose the arrays of the individuals are

Individual 1: $\mathrm{X} \mathrm{Y} \mathrm{Z}$

Individual 2: $\mathrm{YZX}$

Individual 3: $\mathrm{ZX} \mathrm{Y}$

Now let the social welfare function be defined by the following rule: "for every pair of alternatives a vote is taken. That alternative which wins a majority shall preceed the other in the social ranking." $A$ vote taken between $X$ and $Y$ gives a majority to $X$ : thus $X$ shall preceed $Y$. A vote between $Y$ and $Z$ yields a majority to $Y$ : thus $Y$ shall preceed $Z$. But a vote taken between $X$ and $Z$ yields a majority to $Z$ : and $Z$ shall preceed $X$ !

There are three obvious ways to escape this dilemma: 1) We may assume that individual tastes are such that the dilemma does not arise; 2) We may set down an arbitrary social array of alternatives which is independent of any and all individual orderings; or 3) We 
may pick out a particular individual and make the social array correspond to his, to the exclusion of all other considerations.

But 1) is to impose a priori restrictions on individual tastes, 2) is to impose the welfare function on society willy-nilly, and 3) is, of course, dictatorship. The interesting question, then, is whether a social welfare function can be found which is free of these objections. These three points and two additional ones are included in five conditions by which Arrow defines a "satisfactory" social welfare function. These conditions are expressed with a mathematical rigour which need not be reproduced here. Their verbal implications are as follows. Condition 1. There must be three distinct social states and the social welfare function must produce a social ordering no matter how the individuals in the community severally order these three alternatives. Condition 2. The social orderings produced by the social welfare function must respond "positively" to changes in individual values. That is "if one alternative ... rises or remains still in the ordering of every individual... it rises, or at least does not fall in the social ordering." (p. 25)

Condition 3. The social welfare function must be independent of "irrelevant alternatives." Suppose, for example, that individuals order a number of alternatives a priori and a social array of these alternatives is thereby determined. Now let it be discovered that certain among these alternatives must be ruled out as impossible. The social ordering of the remaining alternatives must be unaffected by this discovery.

Condition 4. The social welfare function must not be imposed.

Condition 5. The social welfare function must not be dictatorial.

The force of conditions 1,4 and 5 should be evident from what has already been said. Condition 2 merely implies that, in Arrow's words, what we want is a welfare function, not a "social ill-fare" function. The immediate force of condition 3 is merely that what individuals think about the impossible should not influence social choices made among possible alternatives. There is, however, a more subtle implication to condition 3 to which reference will be made later.

It is to be emphasized that these five conditions provide only minimal "common-sense" restrictions on the nature of the welfare 
function. Not only are they entirely compatible with, say, a liberal democratic philosophy, they are, in fact, much weaker than most of us would be willing to agree to as a minimum. In particular, conditions 4 and 5 do not imply that everyone must, in any sense, "count equally" with everyone else. In point of fact, conditions 4 and 5 would be adequately satisfied provided only two individuals in the community were specified and the social ordering of alternatives somehow determined from their tastes alone. In other words, the five conditions appear to be necessary to (but not sufficient for) social decision processes ranging from egalitarian to something next to dictatorial.

The breadth of possibilities adds great force to Arrow's conclusions. For mild though the five conditions may be, Arrow is able to deduce a rigorous proof of his General Possibility Theorem: "If there are at least three alternatives which the members of society are free to order in any way, then every social welfare function satisfying conditions 2 and 3 , and yielding a social ordering satisfying axioms I and II must be either imposed or dictatorial." (p. 59)

The proof of the general theorem involves the concept of a "decisive" set of individuals. Arrow defines a decisive set as a set of individuals such that when all individuals in the set prefer some alternative $\mathrm{X}$ to some $\mathrm{Y}$, then the social welfare function indicates a social preference of $\mathrm{X}$ to $\mathrm{Y}$. For example, in decision by majority vote any set containing a majority is a decisive set; in a dictatorship any set including the dictator is a decisive set, and so on. Arrow demonstrates, moreover, that the set of all individuals in the society is always a decisive set. I.e., if there is general consensus in the preference of $\mathrm{X}$ to $\mathrm{Y}$, there must always be social preference of $\mathrm{X}$ to $\mathrm{Y}$. This fact guarantees that at least one decisive set always exists, no matter what the form of the social welfare function and no matter what the social decision process may be. It also implies that there must be at least one member in any decisive set: For if nobody thinks $\mathrm{Y}$ is at least as good as $\mathrm{X}$ then everybody must think $\mathrm{X}$ is better than $\mathrm{Y}$ and "everybody" is a decisive set.

Thus for any social welfare function there must be a decisive set. With a finite number of individuals the total number of sub-sets is finite and there must exist a decisive set containing a minimum 
number of individuals. This set must contain at least one individual. Arrow then proves that the minimum decisive set always contains at most one person. But then this one person is a dictator in contradiction to condition 5 .

The General Possibility Theorem (perhaps more properly called the General "Impossibility" 'Theorem) means literally that it is futile, at least in the absence of an interpersonally valid utility measure, to seek a method of combining individual feelings into a social choice in satisfaction of the five weak conditions. Neither market mechanism however competitive, nor legislative processes however elaborately devised, nor indeed any procedure however simply or elegantly arranged will "satisfactorily" reflect personal values in social decisions.

At first sight this may appear as a dismal conclusion to end all dismal conclusions in this dismal science. On the other hand, I think this conclusion has been intuitively recognized by many who have followed the development of welfare economics in the last couple of decades. From this point of view the theorem has merely placed intuition beyond doubt. This being the usual purpose of theorems it is entirely welcome on that score.

The real point, however, is that if we cannot hope for a social welfare function which will satisfy all five conditions, we must ask ourselves which of the five we are willing to relax. Elimination of 4 or 5 is, of course, out of the question. Similarly 2 (that the social welfare function should react "positively" to changes in individual tastes) is apparently inviolate.

As we noticed earlier, however, a social welfare function can be defined provided we rule out those patterns of individual preference which give rise to the paradox of voting. The last half of Arrow's book is devoted to an excellent discussion of this matter. Arrow does not, however, devote a corresponding amount of attention to condition 3 (independence of irrelevant alternatives), but merely points out that it is really condition 3 which stands in the way of employing the technique of summing ordinal individual utility indicators to obtain a social utility, and hence a social ordering. This fact is not immediately apparent in condition 3 , but exhibits itself only when it is shown that the use of aggregate utility numbers can be made compatible with the other four conditions. It follows from the 
general theorem that it must be incompatible with condition 3. This would suggest that some modification of condition 3, perhaps together with a restriction imposed on utility measures might produce interesting results.

In conclusion it will bear special mention that the methodology which Arrow employs makes his book an excellent meeting place for several types of mind. The mathematician or logician who is seeking new applications for his tools and new problems to conquer will find here an excellent example of the rigourous application of his methods to an important social problem. But this is not to say that the non-mathematical social scientist will find the work a bewildering maze of complicated mathematical operations. On the contrary, the mathematical structure is both simple and entirely self-contained. Indeed the volume is admirably adapted to introduce the student of social science to the rigourous application of the axiomatic method. Finally, those who think that exact formalization and mathematical thought are somehow incompatible with appreciation of the broad fundamental problems of social philosophy (and unfortunately there are still many who think so) will find here an argument to the contrary which is, I think, unanswerable.

University of Michigan, Ann Arbor, Michigan Daniel B. SuIts 